\section{- \\ OPEN ACCESS}

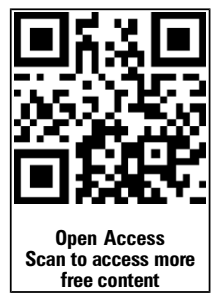

- Additional material is published online only. To view please visit the journal online (http://dx.doi.org/10.1136/ bmjspcare-2013-000593).

${ }^{1}$ Department of Neurosurgery, The University Hospital of Copenhagen, Rigshospitalet, Copenhagen, Denmark ${ }^{2}$ The University Hospitals Center for Health Research (UCSF), Center for Integrated Rehabilitation for Patients with Cancer (CIRE), Copenhagen, Denmark

${ }^{3}$ Department of Clinical Medicine, Section of Neurology, Psychiatry and Sensory Sciences, The University of Copenhagen, Copenhagen K, Denmark ${ }^{4}$ The University Hospital of Copenhagen; Rigshospitalet, Neuroscience Center, Copenhagen, Denmark

\section{Correspondence to} Dr Karin Piil, Department of Neurosurgery, University Hospital of Copenhagen; Rigshospitalet, UCSF 9701, Blegdamsvej 9, Copenhagen 2100, Denmark; Karin.piil@rh.regionh.dk

Received 12 September 2013 Revised 11 April 2014 Accepted 30 April 2014 Published Online First

2 June 2014

\section{CrossMark}

To cite: Piil $\mathrm{K}$, Juhler $\mathrm{M}$ Jakobsen J, et al. BMJ Supportive \& Palliative Care 2016;6:27-34.

\title{
Controlled rehabilitative
}

\section{and supportive care intervention} trials in patients with high-grade gliomas and their caregivers:

\section{a systematic review}

\author{
K Piil, ${ }^{1,2} \mathrm{M}$ Juhler, ${ }^{1,3}$ J Jakobsen, ${ }^{4} \mathrm{M}$ Jarden $^{2}$
}

\section{ABSTRACT}

Background Patients diagnosed with highgrade gliomas experience a varying and complex symptom burden, and face a high mortality rate. As a consequence, patients with high-grade gliomas and their caregivers have imminent and changing rehabilitative and supportive care needs.

Objectives To give a detailed overview of nonpharmacological rehabilitative and supportive care interventions for patients with high-grade gliomas and/or their caregivers, and provide an appraisal of the methodological quality of these studies.

Method PubMed, Cumulative Index of Nursing and Allied Health Literature and Embase were searched for literature published from 1995 to May 2013. Data from eight studies were reviewed for substantive methods and results. Methodological quality was described and assessed using the scoring system for appraising mixed methods research and concomitantly appraising qualitative, quantitative and mixed methods primary studies in mixed study reviews. Results The search yielded 914 unique publications, of which 9 were classified eligible for this review. There is preliminary evidence that cognitive group therapy improves memory skills in patients with high-grade gliomas, early physical training improves functional outcome and massage therapy reduces stress. Patients and caregivers found that telephone follow-up and a specialist nurse function was an effective and useful way to achieve information and support. Finally, psycho-education increased feelings of mastery among caregivers.

Conclusions As evidence is beginning to emerge, there is a need for well-designed longitudinal and randomised controlled trials of non-pharmacological interventions in high-grade glioma patients and their caregivers in order to develop clinical guidelines for supportive and rehabilitative approaches in this unique population.

\section{INTRODUCTION}

High-grade glioma (HGG) is the most malignant type of brain tumour (BT) occurring most frequently in people aged $45-70 .{ }^{1}$ Treated with optimal therapy, the median survival is $12-15$ months with a 5 -year survival of $10 \% .^{2}$ Patients with HGG experience a high symptom burden related to the disease and the surgical procedures and medical treatments. ${ }^{3}{ }^{4}$ Different types of cerebral symptoms and complications may occur, including global cerebral symptoms (fatigue, nausea, headache, confusion) focal symptoms (hemiparesis, seizures, speech difficulties), neurocognitive deficits (impaired attention, concentration difficulties, reduced shortterm memory, personality changes) and emotional symptoms (depression, anxiety, stress). ${ }^{5-9}$ As a consequence, patients with HGG and their caregivers have a number of rehabilitative and supportive care needs. The literature recommends that future intervention studies include individually tailored communication and specialist support ${ }^{10-12}$ to relieve emotional distress in patients with gliomas ${ }^{13}$ and stress among caregivers. ${ }^{14}$ However, little attention has been paid to non-pharmacological rehabilitative and supportive care interventions targeted patients with HGG and/or their caregivers. ${ }^{15}$ A recent Cochrane literature review ${ }^{16}$ was unable to identify any randomised controlled trials (RCT) or controlled clinical trials of multidisciplinary 
rehabilitation interventions in adults after primary BT treatment. ${ }^{16}$ The majority of studies include participants with different subtypes of BT; however, tumour progression varies depending on tumour type. HGG is a rapidly progressive life-threatening disease, while other subgroups of BT progress more slowly. This is supported by investigators who underpin the importance of research on potential differences in needs among the heterogeneous population of patients with BT. ${ }^{17} 18$ Presently, there are no evidence-based rehabilitative and supportive care guidelines that aim to reduce loss of cognition, function and quality of life (QOL). To our knowledge, this is the first systematic literature review that gives a detailed overview of rehabilitative and supportive care intervention studies in patients with HGG and/or their caregivers with a systematic appraisal of the methodological quality of each publication.

\section{METHODS}

This systematic literature search reviews nonpharmacological rehabilitative and supportive care interventions in patients with HGG and their caregivers. Data regarding population, study design, intervention and main results are extracted, synthesised and discussed. The selected publications are appraised for methodological quality. Lastly, implications for future research and conclusions are presented.

\section{Literature search}

The study population includes patients with HGG and/ or their caregivers in non-pharmacological rehabilitative and supportive care intervention studies. Searches were performed in PubMed, Cumulative Index of Nursing and Allied Health Literature (CINAHL) and Embase. Combinations of the following 'Medical Subject Heading' (MeSH) terms and free text terms were used: 'brain cancer', 'brain neoplasms', 'brain tumor', 'highgrade glioma', 'malignant glioma', 'rehabilitation', 'training', 'expectations', 'needs', 'quality of life', 'supportive care', 'intervention', 'program', 'intervention studies', 'caregiver', 'spouse', 'relative' and 'next of kin'. The search strategy is illustrated in figure 1 . The full search strategy including search terms used for each database is available and may be requested by contacting the corresponding author (KP). Reference Manager Software program was used to identify duplicates. The search was limited to English-, Danish-, Norwegian- and Swedish-language publications published from 1995 to May 2013. The systematic literature search was guided by the PRISMA guidelines-preferred reporting items for systematic reviews and meta-analyses developed by Cochrane Collaboration. ${ }^{19}$

\section{Inclusion and exclusion criteria}

Publications included are randomised and nonrandomised controlled trials of unidisciplinary or multidisciplinary non-pharmacological rehabilitative and/or supportive care interventions in patients with
HGG and/or their caregivers. Studies with different subtypes of BT are included only if statistical analysis was performed in the HGG population. Trials studying medical and pharmaceutical treatments are excluded from this review.

\section{Data extraction and quality assessment}

Information regarding the population (sample size, age and attrition), the study design (focus and time of enrolment), the intervention (context, type, mode, duration, frequency, length and adherence) and main results were extracted and systemised using NVivo software program (see online supplementary table S1). ${ }^{20}$ Assessment of methodological quality and scoring of each publication was carried out independently by two researchers (KP and MJA). There was no discrepancy between the raters' scores. The online supplementary table S2 shows an evaluation of the methodological quality of each publication. Methodological quality was assessed using the scoring system for appraising mixed methods research and concomitantly appraising qualitative, quantitative and mixed methods primary studies in mixed study reviews. ${ }^{21}$ Methodological quality for the qualitative studies was assessed for the presence or absence of the following criteria: a qualitative objective or question, appropriate qualitative approach or design or method, a description of the context, a description of participants and justification of sampling, a description of qualitative data and a discussion of researchers' reflexivity collection and analysis. Methodological quality for the quantitative experimental studies was appraised for the presence or absence of the following criteria: an appropriate sequence generation and/or randomisation, allocation concealment and/or blinding, and presentation of complete outcome data and/ or low withdrawal/dropout. For each criterion, the presence or absence is scored 1 or 0 , respectively. The quality score is calculated as a percentage: (number of 'presence' responses divided by the number of relevant criteria) $\times 100$. A further descriptive and detailed critical appraisal of methodological quality is presented.

\section{Ethics}

This systematic review is part of a larger study, which is registered at the Research Ethics Committees at the Regional capital in Copenhagen, Denmark (33460), and the Danish Data Protection Agency (2007-58-0015/30-0758).

\section{RESULTS}

A systematic search was carried out by an information specialist and the primary investigator (KP) in June 2013. The search resulted in 748 hits in PubMed, 114 hits in CINAHL and 52 hits in Embase and is schematically outlined in figure 1 . No duplicates were identified, resulting in 914 unique titles. The titles 
(("Brain Cancer"[tiab] OR "Brain Neoplasms"[Mesh:NoExp] OR "Brain Neoplasms"[tiab] OR "Brain Tumor"[tiab] OR "Brain Tumors"[tiab] OR "Brain Tumour"[tiab] OR "Brain

Tumours"[tiab] OR "High-grade Glioma"[tiab] OR "High-grade Gliomas"[tiab] OR "Malignant Glioma"[tiab] OR "Malignant Gliomas"[tiab]) AND (Rehabilitation[mh] OR Rehabilitation[ti] OR Training[ti] OR "Expectations"[ti] OR Needs*[ti] OR Quality of Life[mh] OR "Quality of Life"[ti] OR "Specialist nurse"[ti] OR Intervention*[ti] OR Program*[ti] OR Intervention Studies[mh] OR Follow-up*[ti] OR

Followup*[ti]) AND ("1995/01/01"[edat] : "2013/05/31"[edat]) AND English[lang]) NOT

(Biological Markers[mh] OR Child[mh] OR Child*[ti] OR Gene[ti] OR Genes[ti] OR Genes[mh] OR Metasta*[ti] OR Pediatric[ti] OR Bevacizumab[ti] OR Temozolomide[ti])

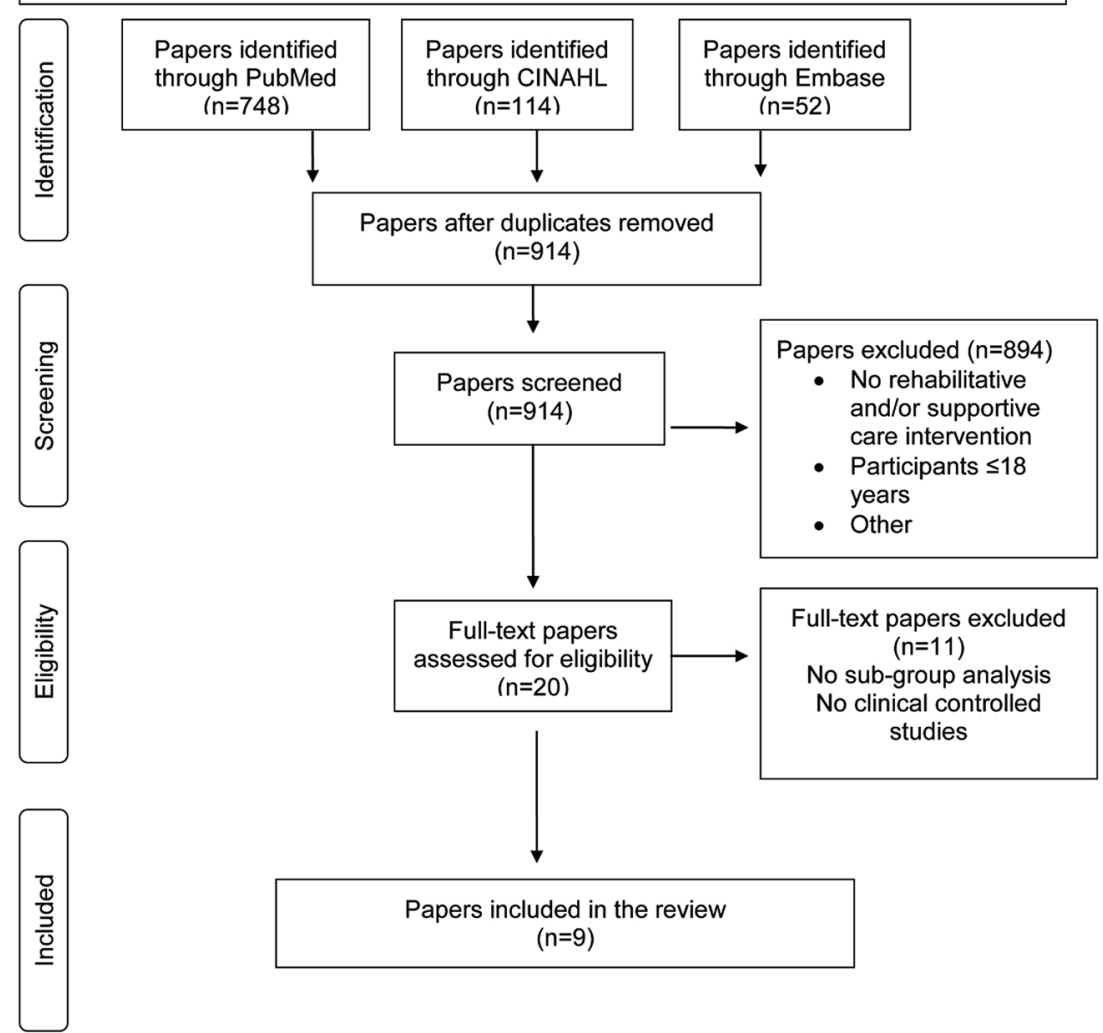

Figure 1 PRISMA flow diagram.

and/or abstracts of the 914 publications were reviewed by the primary investigator (KP), and 894 publications were excluded. Two researchers (KP and MJA) reviewed 20 full manuscripts, which appeared potentially relevant, and 11 were excluded. Nine publications describing eight different interventions met the inclusion criteria. ${ }^{22-30}$ Sardell et al's ${ }^{26}$ mixed BT population study was included because all 45 patients $(\mathrm{n}=43$ HGG; $\mathrm{n}=1$ primitive neuroectodermal tumour; $n=1$ oligoastrocytoma) received primary therapy for high-grade malignancy.

\section{Study characteristics}

The online supplementary table S1 describes populations studied, interventions employed and main results.

The nine publications were published in seven different scientific journals between 2000 and 2012, the majority being published within the last 5 years. The time of patient and/or caregiver enrolment to the interventions varied in the studies, from time of diagnosis to late stages of the disease. Four studies were initiated at time of diagnosis, ${ }^{24} 25272830$ one after hospital discharge ${ }^{23}$ and two studies 15-16 months after diagnosis. $^{22}{ }^{26}$ All interventions were supervised, seven were carried out individually ${ }^{23-30}$ and one intervention was a group intervention. ${ }^{22}$ Six studies were unidisciplinary $^{22} 23 \quad 26-30$ and two were multidisciplinary as they combined physical training with occupational and speech therapy, respectively. ${ }^{24} 25$

\section{Populations studied}

The studies primarily included patients diagnosed with HGG. However, one publication included only caregivers, ${ }^{29}$ and one included both patients and their caregivers. ${ }^{27} 28$ This was the only intervention that continued to intervene with caregivers after the patient died. ${ }^{27} 28$ The age of the patients ranged from 23 to 79 years (mean 55.8). The age of the caregivers was not reported. Sample sizes ranged from 11 to 121 participants (mean 50.8). The demographic characteristics of the participants were included in all but one 
study $^{30}$ and not outlined according to subgroup diagnoses in two studies. 2324

\section{Intervention and outcome characteristics}

Nine publications (eight interventions) reported the results of non-pharmacological rehabilitative and/or supportive care interventions in patients with HGG and/or their caregivers ${ }^{2-30}$ (see online supplementary table S1). Hassler et $a l^{22}$ examined the effect of supervised cognitive group training in patients with HGG, Pace et $a l^{23}$ Geler-Kulcu et $a l^{24}$ and Bartolo et $a l^{25}$ studied the effect of supervised physical training in patients with HGG and Sardell et al, ${ }^{26}$ Spetz et al ${ }^{27} 28$ and Boele et $a l^{29}$ (four publications) tested nurse-led telephone follow-up (NTF), a specialist nurse (SN) function in patients and caregivers and psycho-education in caregivers, respectively. Keir ${ }^{30}$ examined the effect of massage therapy among HGG patients.

Five interventions were conducted in a hospital setting, 2224252930 one of the physical training programmes was carried out as a home programme ${ }^{23}$ and two follow-up studies took place in a combination of a home and hospital setting. ${ }^{26-28}$ The length of the interventions varied considerably, from 2 weeks to comprise the entire disease and treatment trajectory until post bereavement. The duration of the interventions was between 6 and $57 \mathrm{~h}$ (median 27.7); ; $^{24} 2930$ however, it was not specified for three supportive care interventions. ${ }^{26-28}$

Hassler et al's ${ }^{22}$ cognitive group training programme found significant improvement in memory skills measured by verbal memory total learning $(p=0.04)$ and no change in verbal memory delayed recall $(p=0.11)$, psychomotor speed $(p=0.22)$, sustained attention $(p=0.17)$ and verbal fluency $(p=0.29)$. Pace et $a l^{23}$ introduced a home rehabilitation programme with individual physical training and reported clinical improvements for the BT group on KPS and The Bartel Index (BI) measuring Activities of Daily Living. Among these patients, a significant improvement in median BI $(\mathrm{p}=0.001)$ and median KPS $(p=0.001)$ was reported. Subgroup data showed no difference between histological subgroups; however, a functional gain (not specified) was observed in $26 \%$ of the HGG population $(\mathrm{N}=62)$. Geler-Kulcu et al's ${ }^{24}$ trial with physical and occupational therapy compared BT patients postoperatively and patients with stroke. Both groups improved in Motor Assessment Scale (MAS) $(p=0.025 / p=0.002)$, Postural Assessment Scale for Stroke (PASS) $(p=0.002 / p=0.002)$, Berg Balance Scale (BBS) $(p=0.011 / p=0.109)$ and Functional Impairment Measurement (FIM-mobility) $\quad(\mathrm{p}=0.043 / \mathrm{p}=0.007)$. No statistical difference between BT subgroups or between the group of BT patients and stroke patients was observed in any measurement (MAS $p=0.718$, PASS $p=0.817, \quad$ BBS $p=0.109, \quad$ FIM-mobility $\mathrm{p}=0.660)$. Bartolo et $a l^{25}$ found similar results in a physical training and speech therapy intervention in a BT group and a stroke group. All outcome measures improved at postintervention for both groups $(\mathrm{p}<0.001)$, though without statistical differences between groups. A subgroup analysis (meningioma, HGG and stroke) revealed that patients with meningioma achieved better independence in activities of daily living $(p=0.02)$ and mobility $(p=0.04)$ compared with the HGG and stroke group. However, the total FIM scores and other clinical scales revealed no differences between the subgroups. There were four different types of supportive care interventions. ${ }^{26-29}$ Sardell et $a l^{26}$ showed that a monthly and ongoing NTF of patients with HGG was an effective alternative to conventional clinic follow-up with face-to-face consultation. Patient satisfaction was high and the numbers of unscheduled clinic visits were minimised to include acute problems as the NTF assisted the patients with a variety of other problems by telephone. A qualitative study carried out by Spetz et $a l^{27} 28$ included both patients and caregivers and showed that an SN role was a useful way to provide information and support. The SN function increased the family's autonomy and facilitated decision making within the family. The $\mathrm{SN}$ was a resource for the patients, the caregivers and the entire family. Boele et al's ${ }^{29}$ RCT found that a psycho-education intervention in caregivers during a 6-week period increased feelings of mastery $(p=0.021)$. Keir's 4-week massage therapy intervention reduced stress between weeks 2 and $3(p=0.010)$ and improved emotional well-being $(p \leq 0.001)$, BT concerns $(p \leq 0.013)$ and social/family well-being $(\mathrm{p} \leq 0.024)$ at post-testing. All participants were below their baseline stress score 1 week after the massage intervention was completed. ${ }^{30}$

\section{Description of study methodological quality}

Systematic methodological ratings of all the included nine papers, with respect to each relevant criterion, are shown in the online supplementary table S2. Spetz et al's 28 intervention study applied a qualitative approach to explore the effect of the $\mathrm{SN}$ function. It fulfilled the methodological quality criteria by $100 \%$. The study formulated a qualitative objective, applied an appropriate qualitative method, described the context, the participants and made a justification of sampling. Further, appropriate qualitative data collection and analysis was carried out and the researcher's reflexivity was described. Seven intervention studies applied quantitative experimental designs ${ }^{22-26} 29{ }^{30}$; the neurocognitive training study, ${ }^{22}$ the home rehabilitation programme, ${ }^{23}$ the two physical training trials comparing BT patients and stroke patients, ${ }^{24}{ }^{25}$ the evaluation of $\mathrm{NTF}^{26}$ the psycho-education study ${ }^{29}$ and the massage study. ${ }^{30}$ All fulfilled $33.3 \%$ of the quality criteria as they only presented a complete outcome data and/or low dropout rate, except for the psycho-education study that scored $33.3 \%$, as it 
fulfilled the criteria of applying an appropriate randomisation. ${ }^{29}$

Further details regarding the study design, inclusion and exclusion criteria, risk of bias, attrition rates and adverse events are described in the following. All studies were designed as controlled interventions trials. One study published in two papers applied a qualitative design (interview, observation). ${ }^{27} 28$ The remaining seven studies used quantitative designs (RCT, case-control, clinical controlled pilot trials, comparative trials). ${ }^{22-26}{ }^{30}$ All interventions carried out pre-post measurements.

Three interventions were conducted during the early stage of the disease. ${ }^{23-25}$

Statistical methods and $\mathrm{p}$ values were presented in all but two studies. ${ }^{26-28}$ Validated measurement outcomes were applied in all studies, except the qualitative study. ${ }^{27} 28$ All interventions are described in detail in such a way that would allow duplication of the intervention design, except the home training study. ${ }^{23}$

All nine papers defined an inclusion and exclusion criteria. Exclusions were often due to patients/caregivers speaking a language unfamiliar to the investigators or participants being unable to complete the questionnaires. Aside from these systematic exclusions, there was a range of other exclusion criteria related to the patient's disease status and level of physical and cognitive function. The cognitive training study included only patients with a KPS between 80 and $100 \%$, without aphasia and without evidence of tumour recurrence after having received standard oncological treatment. ${ }^{22}$ A high performance status was also a criterion for inclusion in the massage study and the SN follow-up. ${ }^{27} 28{ }^{30}$ Patients with communication or cognitive problems were excluded from the NTF study, ${ }^{26}$ and patient/caregiver groups were excluded if expected survival was less than 3 months. ${ }^{29} 30$ Three studies reduced risk of bias by applying a RCT design ${ }^{29}$ or by including a control group with a one-to-one match. ${ }^{24}{ }^{25}$ One of these studies excluded subtypes of BT in order to maintain a homogeneous group of participants. ${ }^{25}$ The remaining six studies were conducted without a control group. $^{22} 23{ }^{26-28} 30$ Spetz et al ${ }^{27} 28$ concluded that a SN function relies on the person's experience and personal qualities and therefore the function could not be regarded as separate from the person performing it, and Sardell et $a l^{26}$ concluded that the study participants only reflected a selected group of patients.

Attrition rate was low for all studies ranging from $0 \%$ to $12.5 \%$, except Boele et al's psycho-education intervention reported $51.6 \%$ and $32 \%$ attrition in the intervention and control groups, respectively. Boele et al concluded that a six-session intervention with a psychologist and/or four questionnaires at five test points may have been too demanding for the caregivers. Attrition was not reported in the home training study. ${ }^{23}$ An adverse event was reported in the massage study as one patient developed shingles during the intervention and dropped out. $^{30}$ An investigation revealed that this event was unrelated to the massage intervention. Sardell et $\mathrm{al}^{26}$ argued that it was reasonable to assume that HGG patients can be followed up by telephone without adversely affective overall care or survival. Spetz et $a l^{27}$ elaborated on the risk of the $\mathrm{SN}$ being too personally engaged in the relationship with the families that may negatively affect the relationship within the family. Moreover, the authors report that two caregivers abstained from participating in the last interview, which could reflect caregiver dissatisfaction with the intervention. ${ }^{28}$ There were no contraindications to physical training reported. ${ }^{25}$ The great diversity of age, gender and educational background in the small sample size enabled patients to avoid stressful comparisons and competition in the cognitive training programme, and no adverse events were reported from the cognitive training study. ${ }^{22}$ Three studies did not report adverse events. ${ }^{23} 2429$

\section{DISCUSSION}

This systematic review identified the relatively few controlled trials of unidisciplinary or multidisciplinary non-pharmacological rehabilitative and/or supportive care interventions in patients with HGG and/or their caregivers. The eight studies were found to be diverse in purpose, study design and of low methodological quality. Methodological limitations of the reviewed studies include small sample sizes, biases in study design and few subgroup analyses. However, there is initial evidence that cognitive and physical training and psychosocial support benefit HGG patients and/ or their caregivers.

\section{Rehabilitative interventions}

Cognitive training

Hassler et $a l^{22}$ found that a neurocognitive group training programme improved memory skills. Late-stage patients with a KPS of 80-100 were enrolled, and it is yet to be explored whether such a programme can be beneficial to HGG patients with a KPS $\leq 80$. No control group or follow-up measurements after 12 weeks were conducted, leaving the longitudinal effects of cognitive training unknown among HGG patients.

Based on previous research, there is preliminary evidence to suggest that cognitive training among patients with low-grade gliomas (LGG) improves cognitive status. ${ }^{31}$ This RCT study among patients with LGG $(\mathrm{N}=140)$ provides initial evidence of a salutary effect on short-term cognitive complaints and on longer-term cognitive performance and mental fatique. ${ }^{31}$ Further, an internet-based cognitive rehabilitation programme in patients with $\mathrm{BT}$ proved to be a convenient and flexible alternative to in-clinic programmes. ${ }^{32}$ 
Physical training

This systematic review found that early and individually tailored physical training (within weeks after surgery) resulted in significant functional gain for HGG patients. However, functional and neurological gains in the weeks after surgery may be explained by the neurosurgical intervention alone. Geler-Kulcu et al's ${ }^{24}$ findings were inconclusive regarding the effect of physical training and occupational therapy in HGG patients as only nine patients with HGG were included.

No physical training studies reported adverse events as a direct result of testing or training. In two studies, functional outcomes were not significantly different in patients with benign tumours versus malignant tumours or primary versus metastatic BTs or BT patients compared with patients with stroke. ${ }^{23}{ }^{24}$ However, in a third study, meningioma patients achieved a greater functional gain (independence in activities of daily living and mobility) compared with patients with HGG or stroke. ${ }^{25}$ Evidence for the benefit of physical training among HGG patients is emerging. However, this evidence justifies physical training before the adjuvant therapy as two out of three studies included patients before radiotherapy and chemotherapy was administered. $^{24} 25$ Three retrospective studies conclude that patients with BT achieve improved functional outcomes from inpatient rehabilitation. ${ }^{3-35}$ Consistent with this, a comparative study of inpatient rehabilitation among patients with BT and patients with traumatic brain injury patients showed functional gains for both groups. ${ }^{36}$ However, the absolute functional outcomes were smaller in the BT group.

\section{Supportive care interventions}

Psychosocial support

In this systematic review, patient and caregivers' need for psychosocial support was met by applying different psychosocial approaches. Psychosocial support provided by a $\mathrm{SN}$ is of value for both patients and their families, ${ }^{27} 28$ and telephone follow-up is a useful strategy. ${ }^{26}$ Repeated counselling with a psychologist improved QOL issues and mastery of caregivers. ${ }^{29}$ However, high attrition indicates that the programme was too burdensome for the participants. Keir showed that massage therapy reduces the feeling of stress. ${ }^{30} \mathrm{It}$ is not possible to estimate the effect of the massage programme as there was no control group. It is imperative that researchers are familiar with the needs, preferences and interests of these patients and their caregivers in order to design interventions that would result in high adherence and low attrition. Two previous reviews explored the needs of patients with HGG and their family/caregivers, ${ }^{37} 38$ and presently more evidence is being established. ${ }^{39}$

Methodological discussion

The strengths of the reviewed studies were that patients with HGG and/or their caregivers generally accept participation in interventions at various times during the disease and treatment trajectory. Interventions that aim to support the patient and/or caregiver, improve cognitive or functional status and reduce stress have high adherence. However, as participation can be experienced as burdensome and result in high attrition, careful ethical and methodological considerations are required. It has been reported that attrition rates are usually high for supportive care interventions in patients with HGG. ${ }^{40}$ Reasons for attrition were death, medical decline of the patient or high caregiver burden. ${ }^{41}$ High attrition was noted in the RCT that required the participants to respond to four different questionnaires every second month, five times in total. ${ }^{29}$ However, the reason for high attrition in this RCT was not confirmed by the participants. Two studies were matched case-control studies ${ }^{24} 25$ strengthening the evidence of multidisciplinary interventions (combined physical training with either occupational or speech therapy) on functional outcomes. Further, strengths of the reviewed studies include the use of validated measurement outcomes, except in the qualitative study. ${ }^{27} 28$ However, the use of different questionnaires and measurements makes it difficult to compare results.

The limitations of the reviewed studies are that they are heterogeneous in many respects, which makes them non-comparable. Sample sizes are small with some studies including as few as 11 participants. ${ }^{22}$ This questions the validity and reliability of the statistical analyses in the studies. Further study limitations include lack of control groups and longitudinal follow-up periods. Selection bias arises from the exclusion of patients with low-performance status $^{22} 272830$ or cognitive deficit and communication problems. ${ }^{26}$

The scoring system applied for methodological quality needs to be further tested for reliability. ${ }^{21}$ However, a pilot study found it to be a promising appraisal tool ${ }^{42}$ and it is presently used internationally. ${ }^{43}$ Moreover, it provides transparency in how the methodological quality was scored and readers can review the decisions. Finally, an additional descriptive methodological quality appraisal of the included studies supplemented this tool.

\section{CLINICAL AND RESEARCH IMPLICATIONS}

Patients with HGG differ from other cancer diagnoses because of progressive neurological deterioration resulting in changing and complex symptoms along the disease and treatment trajectory. A multidisciplinary approach has the potential to meet the variety of needs emerged from this life-threatening situation. However, cognitive deficits, changes in personality, lack of insight and loss of empathy may impair HGG patients' ability to participate actively in rehabilitative and supportive care programmes. Therefore, it is important that the interventions are tailored to the 
special needs of this group. New approaches, such as internet-based approaches, might have the potential to meet the needs of patients with HGG and their caregivers. ${ }^{44}$ It is well known that decreased QOL is associated with depression among patients with HGG. ${ }^{45}$ However, psychosocial support for patients with HGG has not been the subject of much research, ${ }^{46}$ which is why future research should aim at maintaining QOL. Studies that enrol patients during and after oncological treatments are important as the adjuvant treatment has a negative impact on functional capacity among patients with $\mathrm{HGG}^{47}$ and reduced cardiorespiratory fitness postoperatively. ${ }^{48}$ Further, treatment with glucocorticoids causes muscle atrophy. ${ }^{49}$ From non-cancer clinical populations, it has shown that resistance and endurance physical training can reverse muscle atrophy. ${ }^{50}$ It is therefore interesting to explore whether a similar effect can be achieved for patients with HGG.

\section{CONCLUSION}

As evidence is beginning to emerge, there is a need for well-designed longitudinal and randomised controlled trials of non-pharmacological interventions in patients with HGG and their caregivers. Despite the methodological limitations of the included studies, cognitive training, individual tailored physical training programmes, psychosocial support/education and massage therapy have positive outcomes for both patients and their caregivers, are feasible and so far, without risk for this group. Research is needed to sustain this beginning evidence. To provide stronger evidence, clinical controlled trials with longitudinal designs focusing on cognitive and physical training as well as supportive care are needed.

Acknowledgements The authors would like to thank the information specialist Anders Larsen from The University Hospitals Centre for Health Research (UCSF), Copenhagen, Denmark, for his contribution to the literature search.

Funding The study was supported by grants from The Centre for Integrated Rehabilitation of Cancer Patients (CIRE), a centre established and supported by The Danish Cancer Society and The Novo Nordisk Foundation. Furthermore, by The Neuro Centre at Copenhagen University Hospital; Rigshospitalet in Denmark, The Novo Nordisk Foundation for Clinical Nursing Research, The Capital Regional Research Foundation in Denmark, Torben and Alice Frimodts Foundation, Vera and Flemming Westerbergs Foundation, Hetland Olsen's Foundation and The Research Foundation of Rigshospitalet, Copenhagen University Hospital.

\section{Competing interests None.}

Provenance and peer review Not commissioned; externally peer reviewed.

Open Access This is an Open Access article distributed in accordance with the Creative Commons Attribution Non Commercial (CC BY-NC 3.0) license, which permits others to distribute, remix, adapt, build upon this work noncommercially, and license their derivative works on different terms, provided the original work is properly cited and the use is non-commercial. See: http://creativecommons.org/licenses/by$\mathrm{nc} / 3.0 /$

\section{REFERENCES}

1 Starkweather AR, Sherwood P, Lyon DE, et al. A biobehavioral perspective on depressive symptoms in patients with cerebral astrocytoma. J Neurosci Nurs 2011;43:17-28.

2 Stupp R, Hegi ME, Mason WP, et al. Effects of radiotherapy with concomitant and adjuvant temozolomide versus radiotherapy alone on survival in glioblastoma in a randomised phase III study: 5-year analysis of the EORTC-NCIC trial. Lancet Oncol 2009;10:459-66.

3 Armstrong TS, Cohen MZ, Eriksen LR, et al. Symptom clusters in oncology patients and implications for symptom research in people with primary brain tumors. J Nurs Scholarsh 2004;36:197-206.

4 Kosteljanetz M. Brain tumors-mainly glial tumors in adults. Ugeskr Laeger 2002;164:3017-20.

5 Molassiotis A, Wilson B, Brunton L, et al. Symptom experience in patients with primary brain tumours: a longitudinal exploratory study. Eur J Oncol Nurs 2010;14:410-16.

6 Lovely MP, Miaskowski C, Dodd M. Relationship between fatigue and quality of life in patients with glioblastoma multiforme. Oncol Nurs Forum 1999;26:921-5.

7 Kim YH, Park CK, Kim TM, et al. Seizures during the management of high-grade gliomas: clinical relevance to disease progression. J Neurooncol 2013;113:101-9.

8 Klein M, Postma TJ, Taphoorn MJ, et al. The prognostic value of cognitive functioning in the survival of patients with high-grade glioma. Neurology 2003;61:1796-8.

9 Boele FW, Klein M, Reijneveld JC, et al. Symptom management and quality of life in glioma patients. CNS Oncol 2014;3:37-47.

10 Davies E, Higginson IJ. Communication, information and support for adults with malignant cerebral glioma: a systematic literature review. Support Care Cancer 2003;11:9-21.

11 Diaz JL, Barreto P, Gallego JM, et al. Proper information during the surgical decision-making process lowers the anxiety of patients with high-grade gliomas. Acta Neurochir (Wien) 2009;151:357-62.

12 Lobb EA, Halkett GK, Nowak AK. Patient and caregiver perceptions of communication of prognosis in high grade glioma. J Neurooncol 2011;104:315-22.

13 Rooney AG, McNamara S, Mackinnon M, et al. The frequency, longitudinal course, clinical associations, and causes of emotional distress during primary treatment of cerebral glioma. Neuro Oncol 2013;15:635-43.

14 Keir ST. Levels of stress and intervention preferences of caregivers of brain tumor patients. Cancer Nurs 2007;30: E33-9.

15 Catt S, Chalmers A, Fallowfield L. Psychosocial and supportive-care needs in high-grade glioma. Lancet Oncol 2008;9:884-91.

16 Khan F, Amatya B, Ng L, et al. Multidisciplinary rehabilitation after primary brain tumour treatment. Cochrane Database Syst Rev 2013;1:CD009509.

17 Halkett GK, Lobb EA, Oldham L, et al. The information and support needs of patients diagnosed with high grade glioma. Patient Educ Couns 2010;79:112-19.

18 Parvataneni R, Polley MY, Freeman T, et al. Identifying the needs of brain tumor patients and their caregivers. J Neurooncol 2011;104:737-44.

19 Liberati A, Altman DG, Tetzlaff J, et al. The PRISMA statement for reporting systematic reviews and meta-analyses of studies that evaluate healthcare interventions: Explanation and elaboration. BMJ 2009;339:b2700. 
20 Skorkjær Binderkrantz A, Bøgh Andersen L. Guide to NVivo 9. 1st edn. Hans Reitzels, 2011.

21 Pluye P, Gagnon MP, Griffiths F, et al. A scoring system for appraising mixed methods research and concomitantly appraising qualitative, quantitative and mixed methods primary studies in mixed studies reviews. Int J Nurs Stud 2009;46:529-46.

22 Hassler MR, Elandt K, Preusser M, et al. Neurocognitive training in patients with high-grade glioma: A pilot study. J Neurooncol 2010;97:109-15.

23 Pace A, Parisi C, Di Lelio M, et al. Home rehabilitation for brain tumor patients. J Exp Clin Cancer Res 2007;26:297-300.

24 Geler-Kulcu D, Gulsen G, Buyukbaba E, et al. Functional recovery of patients with brain tumor or acute stroke after rehabilitation: a comparative study. J Clin Neurosci 2009;16:74-8.

25 Bartolo M, Zucchella C, Pace A, et al. Early rehabilitation after surgery improves functional outcome in inpatients with brain tumours. J Neurooncol 2012;107:537-44.

26 Sardell S, Sharpe G, Ashley S, et al. Evaluation of a nurse-led telephone clinic in the follow-up of patients with malignant glioma. Clin Oncol (R Coll Radiol) 2000;12:36-41.

27 Spetz A, Henriksson R, Bergenheim AT, et al. A specialist nurse-function in neurooncology: a qualitative study of possibilities, limitations, and pitfalls. Palliat Support Care 2005;3:121-30.

28 Spetz A, Henriksson R, Salander P. A specialist nurse as a resource for family members to patients with brain tumors: An action research study. Cancer Nurs 2008;31:E18-26.

29 Boele FW, Hoeben W, Hilverda K, et al. Enhancing quality of life and mastery of informal caregivers of high-grade glioma patients: a randomized controlled trial. J Neurooncol 2013;111:303-11.

30 Keir ST. Effect of massage therapy on stress levels and quality of life in brain tumor patients-observations from a pilot study. Support Care Cancer 2011;19:711-5.

31 Gehring K, Sitskoorn MM, Gundy CM, et al. Cognitive rehabilitation in patients with gliomas: a randomized, controlled trial. J Clin Oncol 2009;27:3712-22.

32 Gehring K, Aaronson NK, Taphoorn MJ, et al. Interventions for cognitive deficits in patients with a brain tumor: An update. Expert Rev Anticancer Ther 2010;10:1779-95.

33 Greenberg E, Treger I, Ring H. Rehabilitation outcomes in patients with brain tumors and acute stroke: Comparative study of inpatient rehabilitation. Am J Phys Med Rehabil 2006;85:568-73.

$34 \mathrm{Fu} \mathrm{JB}$, Parsons HA, Shin KY, et al. Comparison of functional outcomes in low- and high-grade astrocytoma rehabilitation inpatients. Am J Phys Med Rehabil 2010;89:205-12.

35 Marciniak CM, Sliwa JA, Heinemann AW, et al. Functional outcomes of persons with brain tumors after inpatient rehabilitation. Arch Phys Med Rehabil 2001;82:457-63.

36 O'Dell MW, Barr K, Spanier D, et al. Functional outcome of inpatient rehabilitation in persons with brain tumors. Arch Phys Med Rehabil 1998;79:1530-4.
37 Sterckx W, Coolbrandt A, Dierckx de Casterle B, et al. The impact of a high-grade glioma on everyday life: A systematic review from the patient's and caregiver's perspective. Eur J Oncol Nurs 2013;17:107-17.

38 Moore G, Collins A, Brand C, et al. Palliative and supportive care needs of patients with high-grade glioma and their carers: A systematic review of qualitative literature. Patient Educ Couns 2013;91:141-53.

39 Piil K, Jarden M, Jakobsen J, et al. A longitudinal, qualitative and quantitative exploration of daily life and need for rehabilitation among patients with high-grade gliomas and their caregivers. BMJ Open 2013;3(7). pii: e003183.

40 Ford E, Catt S, Chalmers A, et al. Systematic review of supportive care needs in patients with primary malignant brain tumors. Neuro Oncol 2012;14:392-404.

41 Meyers FJ, Carducci M, Loscalzo MJ, et al. Effects of a problem-solving intervention (COPE) on quality of life for patients with advanced cancer on clinical trials and their caregivers: Simultaneous care educational intervention (SCEI): Linking palliation and clinical trials. J Palliat Med 2011;14:465-73.

42 Pace R, Pluye P, Bartlett G, et al. Testing the reliability and efficiency of the pilot mixed methods appraisal tool (MMAT) for systematic mixed studies review. Int J Nurs Stud 2012;49:47-53.

43 Polit D, Beck C, eds. Nursing research. Generating and assessing evidence for nursing practice. 9th edn. Wolters Kluwer. Lippincottt Williams \& Wilkins, 2012.

44 Jung Oh H, Ozkaya E, LaRose R. How does online social networking enhance life satisfaction? The relationships among online supportive interaction, affect, perceived social support, sense of community, and life satisfaction. Comput Hum Behav 2014;30:69-78.

45 Cheng JX, Zhang X, Liu BL. Health-related quality of life in patients with high-grade glioma. Neuro Oncol 2009; 11:41-50.

46 Pace A, Metro G, Fabi A. Supportive care in neurooncology. Curr Opin Oncol 2010;22:621-6.

47 Jones LW, Guill B, Keir ST, et al. Exercise interest and preferences among patients diagnosed with primary brain cancer. Support Care Cancer 2007;15:47-55.

48 Jones LW, Friedman AH, West MJ, et al. Quantitative assessment of cardiorespiratory fitness, skeletal muscle function, and body composition in adults with primary malignant glioma. Cancer 2010;116:695-704.

49 Hempen C, Weiss E, Hess CF. Dexamethasone treatment in patients with brain metastases and primary brain tumors: Do the benefits outweigh the side-effects? Support Care Cancer 2002;10:322-8.

50 Horber FF, Hoopeler H, Scheidegger JR, et al. Impact of physical training on the ultrastructure of midthigh muscle in normal subjects and in patients treated with glucocorticoids. J Clin Invest 1987;79:1181-90. 